rhagische Veränderungen oder ein malignes Hirnödem in der Gruppe mit Lyse.

Kommentar: In vielen Situationen stellt sich die Frage, ob eine systemische Lyse auch bei Patienten durchgeführt werden sollte, die einen Verschluss der ACI haben. Insgesamt herrscht die Meinung vor, dass eine systemische Lyse dann nicht sinnvoll ist. Diese Studie spricht jedoch gegen diese Meinung, indem sie zeigt, dass eine systemische Lyse auch bei Patienten mit einem Verschluss der $\mathrm{ACl}$ zu einem besseren Outcome führt. Dies muss natürlich gegen die erhöhte Rate von Blutungen (tödliche und nicht-tödliche) abgewogen werden. Wenn man sich die genaue Verteilung der Patienten in Bezug auf den $\mathrm{mRS}$ ansieht, so ist die Lyse für Patienten mit einem $m R S$ von $<4$ schlechter. Es versterben also vor allem schwer betroffene Patienten mit großen Schlaganfällen, die dann eine Einblutung oder eine Ödementwicklung durch die Reperfusion von schon abgestorbenen Hirnarealen erleiden. Ein anderes Problem dieser Studie ist die Sicherheit, mit der die Diagnose eines Verschlusses der $\mathrm{ACl}$ bei den Patienten gestellt wurde. Eine hohe Rate von Ultraschalldiagnosen in der Kontrollgruppe kann dafür sprechen, dass hier auch einige Patienten mit hochgradigen Stenosen und nicht mit echten ACl-Verschlüssen in die Studie aufgenommen wurden.

Prof. Dr. med. Dr. phil. Stefan Evers

Paciaroni M, Balucani C, Agnelli G et al. Systemic thrombolysis in patients with acute ischemic stroke and Internal Carotid Artery Occlusion: the ICARO study. Stroke 2012; 43: 125-30

\title{
Hilft die Implementierung von Leitlinien auf der Stroke Unit?
}

Die Behandlung auf einer Stroke Unit geht mit einem besseren globalen Outcome der Patienten einher. Allerdings ist unklar, inwieweit spezifische Empfehlungen von Leitlinien für Stroke Units eingehalten werden müssen. Ziel der vorliegenden Studie war es, ein evidenzbasiertes multidisziplinäres Therapiekonzept für Fieber, Hyperglykämie und Schluckstörungen nach einem Schlaganfall zu evaluieren.

E ingeschlossen wurden Patienten mit einem akuten Schlaganfall (ischämischer Infarkt oder Blutung), die sich in einem Krankenhaus vorstellten, in dem eine Stroke Unit vorhanden war. Diese wurden dann randomisiert und entweder nach einem standardisierten, evidenzbasierten Protokoll in einem geschulten Team bezüglich Fiebersenkung, Hyperglykämie und Schluckstörungen behandelt, oder sie erhielten die bisherige Standardtherapie der Stroke Unit bezüglich dieser Bedingungen. Als wichtigste Zielkriterien wurden Tod und funktionelle Abhängigkeit, gemessen mit der Modified Rankin Scale $(\geq 2)$ nach 90 Tagen, gewählt. Patienten und Auswerter der Ergebnisse waren bezüglich der Behandlungsgruppe verblindet.

Insgesamt wurden Daten von 1.696 Patienten erhoben. Die Ergebnisse zeigen, dass die Patienten unabhängig von dem Schweregrad des Schlaganfalls unter einer standardisierten und evidenzbasierten Therapie weniger Todesfälle und weniger funktionelle Abhängigkeit nach 90 Tagen aufwiesen, wenn sie nach dem intensivierten Protokoll behandelt wurden $(42 \%$ vs. $58 \%$; $p=0,002)$. Auch die körperliche Lebensqualität war in der Behandlungsgruppe signifikant besser. Kein Unterschied zeigte sich bei beiden
Gruppen unter anderem im BarthelIndex. Die Autoren folgern daraus, dass die Implementierung eines multidiszi-

Anzeige

$$
\begin{gathered}
\text { Achtung! } \\
\text { Hier muss der } \\
\text { Platzhalter durch } \\
\text { eine Anzeige ersetzt } \\
\text { werden! }
\end{gathered}
$$

besseren Outcome von Patienten nach der Behandlung auf der Stroke Unit führt.

Kommentar: Diese Studie belegt, dass Leitlinien, die evidenzbasiert entstanden sind und in einem multidiziplinären Team umgesetzt werden müssen, auf einer Stroke Unit erfolgreich implementiert werden können. Dies ist keineswegs selbstverständlich, denn bislang ist immer noch nicht klar, welche Elemente einer Behandlung auf der Stroke Unit einen spezifischen Effekt auf das Outcome haben und inwieweit die unspezifische intensive Behandlung und Zuwendung zu dem besseren Outcome führt. Die Studie zeigt auch, dass die häufig nur am Rande beachteten Parameter wie Fieber und Hyperglykämie bei adäquater Behandlung zu einem eindrücklich verbesserten Outcome beitragen können.

Prof. Dr. med. Dr. phil. Stefan Evers
Middleton S, McElduff P, Ward J et al. Implementation of evidence-based treatment protocols to manage fever, hyperglycaemia, and swallowing dysfunction in acute stroke (QASC): a cluster randomised controlled trial. Lancet 2011; 378: 1699-1706 plinären und evidenzbasierten Behandlungsprotokolls bezüglich der oben genannten Parameter zu einem nochmals 